\title{
Analytical Solutions for Two Mixed Initial-Boundary Value Problems Corresponding to Unsteady Motions of Maxwell Fluids through a Porous Plate Channel
}

\author{
Constantin Fetecau $\left(\mathbb{D},{ }^{1}\right.$ Dumitru Vieru $(\mathbb{D})^{2}$ and Ahmed Zeeshan $\mathbb{D D}^{3,4}$ \\ ${ }^{1}$ Section of Mathematics, Academy of Romanian Scientists, Bucharest 050094, Romania \\ ${ }^{2}$ Department of Theoretical Mechanics, Technical University of Iasi, Iasi 700050, Romania \\ ${ }^{3}$ Department of Mathematics \& Statistics, International Islamic University, Islamabad 44000, Pakistan \\ ${ }^{4}$ Department of Mathematics, Faculty of Sciences, King Abdulaziz University, P.O. Box 80203, Jeddah 21589, Saudi Arabia
}

Correspondence should be addressed to Constantin Fetecau; c_fetecau@yahoo.com

Received 13 February 2021; Revised 22 March 2021; Accepted 5 April 2021; Published 26 April 2021

Academic Editor: Jakub Grabski

Copyright (c) 2021 Constantin Fetecau et al. This is an open access article distributed under the Creative Commons Attribution License, which permits unrestricted use, distribution, and reproduction in any medium, provided the original work is properly cited.

\begin{abstract}
Two unsteady motions of incompressible Maxwell fluids between infinite horizontal parallel plates embedded in a porous medium are analytically studied to get exact solutions using the finite Fourier cosine transform. The motion is induced by the lower plate that applies time-dependent shear stresses to the fluid. The solutions that have been obtained satisfy all imposed initial and boundary conditions. They can be easily reduced as limiting cases to known solutions for the incompressible Newtonian fluids. For a check of their correctness, the steady-state solutions are presented in different forms whose equivalence is graphically proved. The effects of physical parameters on the fluid motion are graphically emphasized and discussed. Required time to reach the steady-state is also determined. It is found that the steady-state is rather obtained for Newtonian fluids as compared with Maxwell fluids. Furthermore, the effect of the side walls on the fluid motion is more effective in the case of Newtonian fluids.
\end{abstract}

\section{Introduction}

Exact solutions for initial and boundary value problems are important for many reasons. First of all, they can characterize the behavior of some fluids or solids in various circumstances. Secondly, they can be used as tests to verify numerical schemes which are implemented to study more complex deformation or motion problems. Although the numerical integration of the differential or integral equations can be realized by computers, the accuracy of results is easier established comparing with exact solutions. During the time, many exact solutions have been established for motions of the Newtonian and nonNewtonian fluids in different circumstances (see, for instance, $[1,2]$ for flows between two parallel plates). Generally, they correspond to initial and boundary value problems in which the velocity is given on the boundary although in some practical situations the shear stress is specified on the solid wall.
Relatively in recent studies, Renardy [3] mentioned that boundary conditions on stresses have to be imposed to formulate a well-posed boundary value problem for the flow of a Maxwell fluid between parallel plates. He also considered steady flows of viscoelastic fluids [4] and showed that the Jeffrey model is well-posed in a bounded channel if the extra-stress components are given on the boundary. Actually, in the Newtonian mechanics, the force is the cause and kinematics is the effect and prescribing the shear stress on the boundary is the same to prescribe the force applied to the boundary in order to move it [5]. The first exact solutions for motions of non-Newtonian fluids in which the shear stress is given on the boundary have been obtained by Ting [6] and Waters and King [7], respectively, for flows of second grade or Oldroyd-B fluids over an infinite plate. First exact solutions for unsteady motions of non-Newtonian fluids in which the shear stress is given on a portion of the boundary 
and the velocity on the other part of the boundary seem to be those of Bandelli and Rajagopal [8] for flows of the second grade fluids in cylindrical domains.

In the meantime, different exact solutions for such motions of the non-Newtonian fluids were determined both in rectangular and cylindrical domains (see, for instance, [9-15] and the references therein). General solutions for the motion of Newtonian fluids between parallel plates in which the shear stress is given on a part of the boundary were recently determined by Javaid et al. [16]. Other exact solutions for such motions of the incompressible Newtonian fluids with pressuredependent viscosity were recently provided by Danish et al. [17]. Exact expressions for the steady-state solutions corresponding to oscillatory motions of the Maxwell fluids in long tubes of rectangular or triangular cross section were recently obtained by Wang et al. [18] and Sun et al. [19], respectively. Starting solutions for nonsteady flows of the Maxwell fluids induced by a moving wall through a porous plate channel were recently provided by Fetecau et al. [20]. An interesting study concerning the Maxwell fluid flow through porous plate was developed by Shahid [21]. To the best of our knowledge, closedform expressions for starting solutions corresponding to mixed initial-boundary value problems of the Maxwell fluids moving in a porous medium lack in the literature.

The purpose of this note is to provide analytical expressions for two unsteady motions of incompressible Maxwell fluids through a horizontal porous plate channel. The fluid motion is generated by the lower plate that applies time-dependent shear stresses to the fluid. Obtained solutions fulfill all imposed initial and boundary conditions and are easily reduced to known solutions for the Newtonian fluids. For validation, the steady-state solutions are presented in different forms and their equivalence is graphically proved. Effects of pertinent parameters on the fluid movement are graphically underlined and interpreted. The required time to reach the steady-state is graphically determined. It is found that the steady-state is later obtained for Maxwell fluids as compared with Newtonian fluids. Furthermore, the effect of the side walls on the fluid motion is more effective on Newtonian fluids than the Maxwell fluids.

\section{Statement of the Problem}

Let us consider an incompressible upper-convected Maxwell (UCM) fluid between two infinite horizontal parallel plates embedded in a porous medium as illustrated in Figure 1. [22]:

The constitutive equations of such a fluid are as follows

$$
\mathbf{T}=-p \mathbf{I}+\mathbf{S}, \quad \mathbf{S}+\lambda\left(\frac{d \mathbf{S}}{d t}-\mathbf{L} \mathbf{S}-\mathbf{S L}^{T}\right)=\mu\left(\mathbf{L}+\mathbf{L}^{T}\right),
$$

where $\boldsymbol{T}$ is the Cauchy stress tensor, $S$ is the extra-stress tensor, $-p \mathbf{I}$ is the constitutively indeterminate part of the stress due to the constraint of incompressibility, $\lambda$ is the relaxation time, $\mu$ is the fluid viscosity, and $L$ is the gradient of the velocity field $v$. In the following, we establish exact solutions for two mixed initial-boundary value problems corresponding to unsteady motions of incompressible UCM fluids into abovementioned domain. All results will be reported to a convenient Cartesian coordinate system having the $y$-axis perpendicular to plates.

Since the plates are infinite in extent, all physical entities which characterize the fluid motion in such a domain are functions of $y$ and $t$ only [23]. Consequently, for such motions of the incompressible UCM fluids, we can look for a velocity field of the form as follows:

$$
v=v(y, t)=u(y, t) \mathbf{e}_{x},
$$

where $\mathbf{e}_{x}$ is the unit vector along the $x$-direction. We also suppose that the extra-stress tensor $S$, as well as the fluid velocity $v$, depends on $y$ and $t$ only.

Replacing the above velocity field in the constitutive equation (1) and bearing in mind the fact that the fluid was at rest up to the moment $t=0$, i.e.,

$$
v(y, 0)=\mathbf{0}, \quad \mathbf{S}(y, 0)=\mathbf{0},
$$

it can be proved that the components $S_{x z}, S_{y z}, S_{y y}$, and $S_{z z}$ of $S$ are zero and [20]

$$
\begin{aligned}
\tau(y, t)+\lambda \frac{\partial \tau(y, t)}{\partial t} & =\mu \frac{\partial u(y, t)}{\partial y}, \quad \sigma(y, t)+\lambda \frac{\partial \sigma(y, t)}{\partial t} \\
& =2 \lambda \tau(y, t) \frac{\partial u(y, t)}{\partial y},
\end{aligned}
$$

where $\tau(y, t)=S_{x y}(y, t)$ and $\sigma(y, t)=S_{x x}(y, t)$ are the non-null components of $S$.

Let us now assume that at the moment $t=0^{+}$, the lower plate begins to apply to the fluid a shear stress $\tau(0, t)$ having one of the forms as follows:

$$
\begin{aligned}
\tau(0, t) & =S_{1}\left\{t-\lambda\left[1-\exp \left(-\frac{t}{\lambda}\right)\right]\right\}, \quad \tau(0, t) \\
& =S_{2} \frac{\sin (\omega t)-\lambda \omega \cos (\omega t)}{(\lambda \omega)^{2}+1}+\frac{\lambda \omega S_{2}}{(\lambda \omega)^{2}+1} \exp \left(-\frac{t}{\lambda}\right),
\end{aligned}
$$

where $S_{1}$ and $S_{2}$ are dimensional constants. In the case $\lambda \longrightarrow 0$, corresponding to Newtonian fluids, the above relations take the simple forms as follows:

$$
\tau(0, t)=S_{1} t \text {, respectively } \tau(0, t)=S_{2} \sin (\omega t) .
$$

Boundary conditions of same forms (6), but for the velocity components, have been recently used by Wang and Liu (see Section 3 in [24]) for the helical flow of generalized Maxwell fluids.

Due to the shear, the fluid begins to move and, in the absence of a pressure gradient in the $x$-direction, the balance of linear momentum reduces to the partial differential equation as follows [25]:

$$
\rho \frac{\partial u(y, t)}{\partial t}=\frac{\partial \tau(y, t)}{\partial y}+R(y, t)
$$




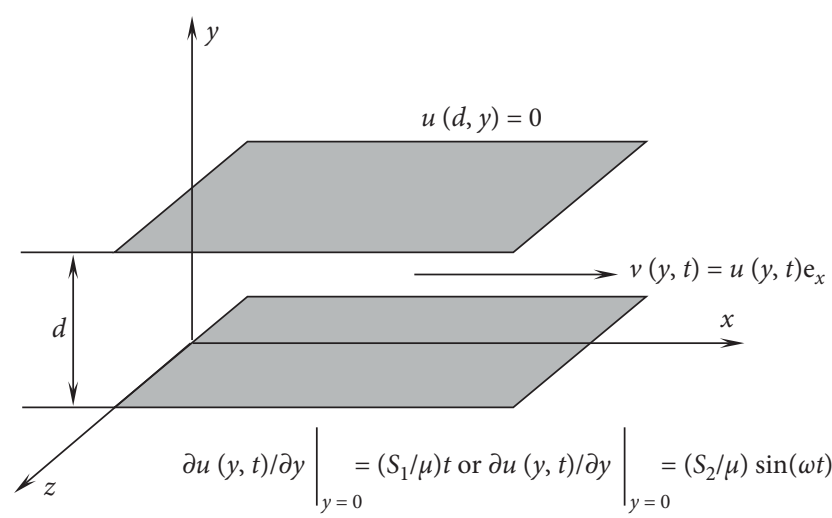

Figure 1: Geometry of the flow.

where $R(y, t)$ is Darcy's resistance which for such fluids has to satisfy the following relation:

$$
R(y, t)+\lambda \frac{\partial R(y, t)}{\partial t}=-\frac{\mu \phi}{k} u(y, t) .
$$

In the above relations, $\rho$ is the fluid density while $\phi$ and $k$ are the porosity and the permeability, respectively, of the porous medium. The continuity equation is identically satisfied.

Eliminating $\tau(y, t)$ between equations (4) and (7) and using equation (8), one attains the following dimensional governing equation for the velocity field:

$$
\begin{gathered}
\lambda \frac{\partial^{2} u(y, t)}{\partial t^{2}}+\frac{\partial u(y, t)}{\partial t}=v \frac{\partial^{2} u(y, t)}{\partial y^{2}}-\frac{\nu \phi}{k} u(y, t) ; \\
0<y<d, \quad t>0,
\end{gathered}
$$

in which $\nu=\mu / \rho$ is the kinematic viscosity and $d$ is the distance between plates.

The appropriate initial and boundary conditions are

$$
\begin{aligned}
u(y, 0) & =0,\left.\quad \frac{\partial u(y, t)}{\partial t}\right|_{t=0}=0 ; \quad 0 \leq y \leq d, \\
\tau(0, t)+\lambda \frac{\partial \tau(0, t)}{\partial t} & =\left.\mu \frac{\partial u(y, t)}{\partial y}\right|_{y=0} \\
& =S_{1} t, \quad u(d, t)=0 \quad \text { if } \quad t>0,
\end{aligned}
$$

for the first motion, and the initial conditions (10) together with the boundary conditions are as follows:

$$
\begin{gathered}
\tau(0, t)+\lambda \frac{\partial \tau(0, t)}{\partial t}=\left.\mu \frac{\partial u(y, t)}{\partial y}\right|_{y=0}=S_{2} \sin (\omega t), \\
u(d, t)=0 \quad \text { if } \quad t>0,
\end{gathered}
$$

in the case of the second motion. It is worth to point out the fact that solving the ordinary differential equations (11) and (12) with respect to $\tau(0, t)$ when the initial condition $\tau(0,0)=0$ resulting from equation (3) is satisfied, and equality (5) is recovered.

As soon as the velocity field $u(y, t)$ is known, the shear and normal stresses $\tau(y, t)$ and $\sigma(y, t)$ can be determined solving the governing equation (4) with the initial conditions as follows:

$$
\tau(y, 0)=0, \quad \sigma(y, 0)=0 ; \quad 0 \leq y \leq d .
$$

However, in order to avoid repetition, we shall here determine the shear stress $\tau(y, t)$ only. It is necessary, for instance, to provide the frictional force per unit area exerted by the fluid on the stationary plate.

\section{Solution}

In the following, the finite Fourier cosine transform, defined by equation (A.1) from Appendix, is used to determine analytical expressions for the dimensionless velocity and shear stress fields corresponding to the two unsteady motions of the incompressible UCM fluids through a porous plate channel.

3.1. Case $\tau(0, t)=S_{1}\{t-\lambda[1-\exp (-t / \lambda)]\}$. In order to determine solutions which are independent of the flow geometry, let us introduce the following nondimensional variables and functions:

$$
\begin{aligned}
& y^{*}=\frac{y}{d}, \quad t^{*}=\frac{v}{d^{2}} t, \quad u^{*}=\frac{\mu \nu}{d^{3} S_{1}} u, \\
& \tau^{*}=\frac{v}{d^{2} S_{1}} \tau, \quad \sigma^{*}=\frac{v}{d^{2} S_{1}} \sigma .
\end{aligned}
$$

Introducing the above nondimensional entities in equations (9)-(11) and giving up the star notation, the next mixed initial-boundary value problem

$$
\begin{gathered}
\mathrm{We} \frac{\partial^{2} u(y, t)}{\partial t^{2}}+\frac{\partial u(y, t)}{\partial t}=\frac{\partial^{2} u(y, t)}{\partial y^{2}} \\
-K u(y, t) ; \quad 0<y<1, \quad t>0, \\
u(y, 0)=0,\left.\quad \frac{\partial u(y, t)}{\partial t}\right|_{t=0}=0 \quad \text { for } \quad 0 \leq y \leq 1, \\
\left.\frac{\partial u(y, t)}{\partial y}\right|_{y=0}=t, \quad u(1,0)=0 \quad \text { if } \quad t>0,
\end{gathered}
$$

is obtained. In equation (15), the Weissenberg number We and the dimensionless porosity parameter $K$ are defined by the equalities as follows:

$$
\mathrm{We}=\frac{\lambda V}{d}, \quad K=\frac{\phi}{k} d^{2},
$$

where $V=\nu / d$ is a characteristic velocity. The Weissenberg number We represents the ratio of the relaxation time $\lambda$ and the characteristic time $t_{0}=d / V$.

The dimensionless forms of the ordinary differential equation (4) are 


$$
\begin{aligned}
\mathrm{We} \frac{\partial \tau(y, t)}{\partial t}+\tau(y, t) & =\frac{\partial u(y, t)}{\partial y} \\
\mathrm{We} \frac{\partial \sigma(y, t)}{\partial t}+\sigma(y, t) & =2 \alpha \tau(y, t) \frac{\partial u(y, t)}{\partial y} ; \quad 0<y<1, \quad t>0,
\end{aligned}
$$

where $\alpha=\lambda d^{2} S_{1} /(\mu \nu)$. The corresponding initial conditions are

$$
\mathrm{We} \frac{d^{2} u_{F n}(t)}{d t^{2}}+\frac{d u_{F n}(t)}{d t}+\left(\mu_{n}^{2}+K\right) u_{F n}(t)=-t ; \quad t>0, \quad n=1,2,3 \ldots
$$

with the initial conditions

$$
u_{F n}(0)=0,\left.\quad \frac{d u_{F n}(t)}{d t}\right|_{t=0}=0 ; \quad n=1,2,3 \ldots
$$

$$
\tau(y, 0)=0 \text { and } \sigma(y, 0)=0 ; \quad 0 \leq y \leq 1 .
$$

Multiplying equation (15) with $\cos \left(\mu_{n} y\right)$, where $\mu_{n}=(2 n+1) \pi / 2$, integrating the result from zero to one, and bearing in mind the initial and boundary conditions (16) and (17) and equalities (A.1) and (A.2) from Appendix, it results that the finite Fourier cosine transform $u_{F n}(t)$ of $u(y, t)$ has to satisfy the ordinary differential equation as follows:

$$
u_{F n}(t)=\frac{1}{a_{n}}\left\{-t+\frac{1}{a_{n}}+\frac{\left(a_{n}+r_{1 n}\right) e^{r_{2 n} t}-\left(a_{n}+r_{2 n}\right) e^{r_{1 n} t}}{\left(r_{2 n}-r_{1 n}\right) a_{n}}\right\} ; \quad n=1,2,3 \ldots
$$

where $a_{n}=\mu_{n}^{2}+K$ and $r_{1 n, 2 n}=\left[-1 \pm \sqrt{1-4 \mathrm{We} a_{n}}\right] / 2 \mathrm{We}$. On the basis of equality (A.1),it results that the
The solution of equation (21) with the initial conditions (22) is given by the relation as follows:

$$
u(y, t)=2 \sum_{n=0}^{\infty}\left\{-t+\frac{1}{a_{n}}+\frac{\left(a_{n}+r_{1 n}\right) e^{r_{2 n} t}-\left(a_{n}+r_{2 n}\right) e^{r_{1 n} t}}{\left(r_{2 n}-r_{1 n}\right) a_{n}}\right\} \frac{\cos \left(\mu_{n} y\right)}{a_{n}} ; \quad 0<y<1, \quad t>0
$$

or equivalently (see the equality (A.3) from Appendix)

$$
u(y, t)=(y-1) t+2 t K \sum_{n=0}^{\infty} \frac{\cos \left(\mu_{n} y\right)}{a_{n} \mu_{n}^{2}}+2 \sum_{n=0}^{\infty}\left[1+\frac{\left(a_{n}+r_{1 n}\right) e^{r_{2 n} t}-\left(a_{n}+r_{2 n}\right) e^{r_{1 n} t}}{r_{2 n}-r_{1 n}}\right] \frac{\cos \left(\mu_{n} y\right)}{a_{n}^{2}} .
$$

Direct computations clearly show that $u(y, t)$ given by equality (25) satisfies both the governing equation (15) and all imposed initial and boundary conditions. The dimensionless volume flux across a plane normal to the $x$ axis and per unit width of this plane is given by the relation as follows:

$$
Q=Q(t)=-\frac{t}{2}+2 t K \sum_{n=0}^{\infty} \frac{(-1)^{n}}{\mu_{n}^{3}\left(\mu_{n}^{2}+K\right)}+2 \sum_{n=0}^{\infty}\left[1+\frac{\left(a_{n}+r_{1 n}\right) e^{r_{2 n} t}-\left(a_{n}+r_{2 n}\right) e^{r_{1 n} t}}{r_{2 n}-r_{1 n}}\right] \frac{(-1)^{n}}{\left(\mu_{n}^{2}+K\right)^{2}}
$$

For $t=0$, as expected, $Q$ becomes zero.

In order to determine the dimensionless frictional force per unit area exerted by the fluid on the stationary plate, the corresponding shear stress $\tau(y, t)$ has to be known.
Replacing the expression of $u(y, t)$ from equality (25) in (19) and solving the obtained equation with the initial condition (20), it results that $\tau(y, t)$ is given by the following expression: 


$$
\begin{aligned}
\tau(y, t)= & \left\{t-W e\left[1-\exp \left(-\frac{t}{W e}\right)\right]\right\}\left[1-2 K \sum_{n=0}^{\infty} \frac{\sin \left(\mu_{n} y\right)}{\mu_{n}\left(\mu_{n}^{2}+K\right)}\right] \\
& -2 \sum_{n=0}^{\infty}\left[1+\frac{\left(a_{n}+r_{1 n}\right)\left(W e r_{1 n}+1\right) e^{r_{2 n} t}-\left(a_{n}+r_{2 n}\right)\left(W e r_{2 n}+1\right) e^{r_{1 n} t}}{\left(r_{2 n}-r_{1 n}\right)\left(W e r_{1 n}+1\right)\left(W e r_{2 n}+1\right)}\right] \frac{\mu_{n} \sin \left(\mu_{n} y\right)}{\left(\mu_{n}^{2}+K\right)^{2}} \\
& +2 \exp \left(-\frac{t}{W e}\right) \sum_{n=0}^{\infty}\left[1+\frac{\left(a_{n}+r_{1 n}\right)\left(W e r_{1 n}+1\right)-\left(a_{n}+r_{2 n}\right)\left(W e r_{2 n}+1\right)}{\left(r_{2 n}-r_{1 n}\right)\left(W e r_{1 n}+1\right)\left(W e r_{2 n}+1\right)}\right] \frac{\mu_{n} \sin \left(\mu_{n} y\right)}{\left(\mu_{n}^{2}+K\right)^{2} .}
\end{aligned}
$$

The motion whose solutions have been determined in this subsection is unsteady and remains unsteady. It is clear that at large enough values of the time $t$, the fluid motion can be described by the long time solutions:

$$
\begin{aligned}
& u_{L t}(y, t)=(y-1) t+2 t K \sum_{n=0}^{\infty} \frac{\cos \left(\mu_{n} y\right)}{\mu_{n}^{2}\left(\mu_{n}^{2}+K\right)}+2 \sum_{n=0}^{\infty} \frac{\cos \left(\mu_{n} y\right)}{\left(\mu_{n}^{2}+K\right)^{2}}, \\
& \tau_{L t}(y, t)=(t-W e)\left[1-2 K \sum_{n=0}^{\infty} \frac{\sin \left(\mu_{n} y\right)}{\mu_{n}\left(\mu_{n}^{2}+K\right)}\right]-2 \sum_{n=0}^{\infty} \frac{\mu_{n} \sin \left(\mu_{n} y\right)}{\left(\mu_{n}^{2}+K\right)^{2}} .
\end{aligned}
$$

As expected, the dimensionless velocity and shear stress fields $u_{N}(y, t)$ and $\tau_{N}(y, t)$ corresponding to incompressible Newtonian fluids performing the same motion, namely,

$$
\begin{aligned}
& u_{N}(y, t)=(y-1) t+2 t K \sum_{n=0}^{\infty} \frac{\cos \left(\mu_{n} y\right)}{\mu_{n}^{2}\left(\mu_{n}^{2}+K\right)}+2 \sum_{n=0}^{\infty}\left[1-e^{-\left(\mu_{n}^{2}+K\right) t}\right] \frac{\cos \left(\mu_{n} y\right)}{\left(\mu_{n}^{2}+K\right)^{2}}, \\
& \tau_{N}(y, t)=t-2 t K \sum_{n=0}^{\infty} \frac{\sin \left(\mu_{n} y\right)}{\mu_{n}\left(\mu_{n}^{2}+K\right)}-2 \sum_{n=0}^{\infty}\left[1-e^{-\left(\mu_{n}^{2}+K\right) t}\right] \frac{\mu_{n} \sin \left(\mu_{n} y\right)}{\left(\mu_{n}^{2}+K\right)^{2}},
\end{aligned}
$$

are immediately obtained making $\mathrm{We} \longrightarrow 0$ in equations (25) and (27). Of course, the last solutions are in agreement with the solutions obtained in equalities (29) and (31) in [16] where the boundary condition (8) has been taken with a changed sign. The corresponding volume flux $Q_{N}$ can be immediately obtained making $\mathrm{We}=0$ in the general expression of $Q$. In the absence of the porous effects, the corresponding solutions can be determined making $K \longrightarrow 0$ into the above relations.

From equalities (28) and (30), it clearly results that the long time velocity fields $u_{L t}(y, t)$ and $u_{N L t}(y, t)$ corresponding to the incompressible UCM and Newtonian fluids, respectively, are identical. Consequently, for large enough values of the time $t$, the dimensionless velocity field $u_{L t}(y, t)$ corresponding to incompressible UCM fluids can be approximated with that of Newtonian fluids. Of course, bearing in mind known results from the existing literature, this is not a surprise. However, this property is not valid for the corresponding shear stress fields. The corresponding frictional forces per unit area exerted by the fluid on the upper plate can be immediately determined making $y=1$ in any one of the relations (27), (29), or (31).

3.2. Case $\tau(0, t)=S_{2}\{\sin (\omega t)-\lambda \omega[\cos (\omega t)-\exp (-t / \lambda)]\}$ $/\left[(\lambda \omega)^{2}+1\right]$. Introducing the next nondimensional variables, functions, and parameters such as

$$
\begin{aligned}
& y^{*}=\frac{y}{d}, \quad t^{*}=\frac{v}{d^{2}} t, \quad u^{*}=\frac{\mu}{d S_{2}} u, \\
& \tau^{*}=\frac{\tau}{S_{2}}, \quad \sigma^{*}=\frac{\sigma}{S_{2}}, \quad \omega^{*}=\frac{d^{2}}{\nu} \omega
\end{aligned}
$$

in equality (9) and again dropping out the star notation, one obtains for the velocity field $u_{s}(y, t)$ corresponding to this motion a dimensionless governing equation of the same form as (15) with the initial condition (16). The corresponding boundary conditions are as follows: 


$$
\left.\frac{\partial u_{s}(y, t)}{\partial y}\right|_{y=0}=\sin (\omega t), \quad u_{s}(1, t)=0 \quad \text { for } \quad t>0 .
$$

Dimensionless governing equations for $\tau_{s}(y, t)$ and $\sigma_{s}(y, t)$ and the corresponding initial conditions are also given by equations (19) and (20) where the new constant $\alpha=\lambda S_{2} / \mu$.

The solution of this new problem with initial and mixed boundary conditions can be determined following the same way as before. To avoid repetition, we here present the final results only. As usually, the dimensionless starting solutions $u_{s}(y, t)$ and $\tau_{s}(y, t)$ of such a motion that become steady in time can be presented under the forms as follows:

$u_{s}(y, t)=u_{s p}(y, t)+u_{s t}(y, t), \quad \tau_{s}(y, t)=\tau_{s p}(y, t)+\tau_{s t}(y, t)$,

in which their steady-state (permanent or long time) and transient components are given by the next relations as follows:

$$
\begin{aligned}
& u_{s p}(y, t)=(y-1) \sin (\omega t)+2 \sum_{n=0}^{\infty} \frac{b_{n} \sin (\omega t)+\omega \mu_{n}^{2} \cos (\omega t)}{a_{n}^{2}+\omega^{2}} \frac{\cos \left(\mu_{n} y\right)}{\mu_{n}^{2}}, \\
& \tau_{s p}(y, t)= \frac{\sin (\omega t)-\omega \mathrm{We} \cos (\omega t)}{(\omega \mathrm{We})^{2}+1}, \\
&-2 \sum_{n=0}^{\infty} \frac{\left(b_{n}+W e \omega^{2} \mu_{n}^{2}\right) \sin (\omega t)+\omega\left(\mu_{n}^{2}-W e b_{n}\right) \cos (\omega t)}{\left(a_{n}^{2}+\omega^{2}\right)\left[(\omega \mathrm{We})^{2}+1\right]} \frac{\sin \left(\mu_{n} y\right)}{\mu_{n}}, \\
& u_{s t}(y, t)=2 \omega \sum_{n=0}^{\infty} \frac{\left(a_{n}+r_{1 n}\right) \mathrm{e}^{r_{2 n} t}-\left(a_{n}+r_{2 n}\right) \mathrm{e}^{r_{1 n} t}}{\left(r_{2 n}-r_{1 n}\right)\left(a_{n}^{2}+\omega^{2}\right)} \cos \left(\mu_{n} y\right), \\
& \tau_{s t}(y, t)= \frac{\omega \exp (-t / \mathrm{We})}{(\omega \mathrm{We})^{2}+1}\left[\mathrm{We}-2 \sum_{n=0}^{\infty} \frac{\mu_{n}^{2}-\mathrm{We}_{n}}{a_{n}^{2}+\omega^{2}} \frac{\sin \left(\mu_{n} y\right)}{\mu_{n}}\right] \\
&-2 \omega \sum_{n=0}^{\infty} \frac{\left(a_{n}+r_{1 n}\right)\left(\mathrm{Wer}_{1 n}+1\right) \mathrm{e}^{r_{2 n} t}-\left(a_{n}+r_{2 n}\right)\left(\mathrm{Wer}_{2 n}+1\right) \mathrm{e}^{r_{1 n} t}}{\left(r_{2 n}-r_{1 n}\right)\left(a_{n}^{2}+\omega^{2}\right)\left(\mathrm{Wer}_{1 n}+1\right)\left(\mathrm{We}_{2 n}+1\right)} \mu_{n} \sin \left(\mu_{n} y\right) \\
&+2 \omega \exp \left(-\frac{t}{\mathrm{We}}\right) \sum_{n=0}^{\infty} \frac{\left(a_{n}+r_{1 n}\right)\left(\mathrm{Wer}_{1 n}+1\right)-\left(a_{n}+r_{2 n}\right)\left(\mathrm{Wer}_{2 n}+1\right)}{\left(r_{2 n}-r_{1 n}\right)\left(a_{n}^{2}+\omega^{2}\right)\left(\mathrm{Wer}_{1 n}+1\right)\left(\mathrm{Wer}_{2 n}+1\right)} \mu_{n} \sin \left(\mu_{n} y\right),
\end{aligned}
$$

where $b_{n}=a_{n} K+\left(1-a_{n} W e\right) \omega^{2}$.

Direct computations show that $u_{s}(y, t)$ and $\tau_{s}(y, t)$ given by equation (34) satisfy both the corresponding governing equations and all imposed initial and boundary conditions. Sometime after the motion initiation, the fluid moves according to these solutions. After this time, when the transients disappear or can be neglected, the fluid motion is characterized by the steady-state solutions given by equations (35) and (36). It is worth pointing out that the steadystate components of $u_{s}(y, t)$ and $\tau_{s}(y, t)$ can be also presented in the simpler forms as follows:

$$
u_{s p}(y, t)=\operatorname{Im}\left\{\frac{\sinh [(y-1) \sqrt{\gamma}]}{\sqrt{\gamma} \cosh (\sqrt{\gamma})} e^{i \omega t}\right\}, \quad \tau_{s p}(y, t)=\operatorname{Im}\left\{\frac{\cosh [(y-1) \sqrt{\gamma}]}{(1+i \omega \mathrm{We}) \cosh (\sqrt{\gamma})} e^{i \omega t}\right\},
$$


where $\gamma=K-\mathrm{We} \omega^{2}+i \omega, i$ is the imaginary unit and Im denotes the imaginary part of that which follows. The equivalence of the steady-state solutions given by the equalities (35) and (36) with those from the relations (39) is graphically proved in Figures 2 and 3.
The dimensionless steady-state and transient solutions corresponding to the motion of the incompressible Newtonian fluids induced by the lower plate that applies a oscillatory shear stress of form (6) to the fluid, namely,

$$
\begin{aligned}
& u_{N s p}(y, t)=(y-1) \sin (\omega t)+2 \sum_{n=0}^{\infty} \frac{\left(a_{n} K+\omega^{2}\right) \sin (\omega t)+\omega \mu_{n}^{2} \cos (\omega t)}{a_{n}^{2}+\omega^{2}} \frac{\cos \left(\mu_{n} y\right)}{\mu_{n}^{2}}, \\
& \tau_{N s p}(y, t)=\sin (\omega t)-2 \sum_{n=0}^{\infty} \frac{\left(a_{n} K+\omega^{2}\right) \sin (\omega t)+\omega \mu_{n}^{2} \cos (\omega t)}{a_{n}^{2}+\omega^{2}} \frac{\sin \left(\mu_{n} y\right)}{\mu_{n}}, \\
& u_{N s t}(y, t)=-2 \omega \sum_{n=0}^{\infty} \frac{\cos \left(\mu_{n} y\right)}{\left(\mu_{n}^{2}+K\right)^{2}+\omega^{2}} e^{-\left(\mu_{n}^{2}+K\right) t} \\
& \tau_{N s t}(y, t)=2 \omega \sum_{n=0}^{\infty} \frac{\mu_{n} \sin \left(\mu_{n} y\right)}{\left(\mu_{n}^{2}+K\right)^{2}+\omega^{2}} \mathrm{e}^{-\left(\mu_{n}^{2}+K\right) t}
\end{aligned}
$$

are immediately obtained making $\mathrm{We} \longrightarrow 0$ in equations (35)-(38). As expected, the expressions of $u_{N s p}(y, t)$ and $u_{N s t}(y, t)$ given by equations (40) and (42), respectively, are also in accordance with the results obtained by Javaid et al. (see equalities (40) and (41) in [16]) where the corresponding boundary condition (8) has been taken with an opposite sign and a different normalization was considered.

\section{Some Numerical Results and Discussion}

Exact solutions for two dimensionless mixed initialboundary value problems corresponding to unsteady motions of incompressible UCM fluids through a porous plate channel have been established in a simple way using the finite Fourier cosine transform only. Their limiting expressions, when the relaxation time $\lambda$ and therefore the Weissenberg number We tends to zero, correspond to motions of the incompressible Newtonian fluids induced by the lower plate that applies ramp type or oscillating shear stresses to the fluid. The steady-state solutions $u_{s p}(y, t)$ and $\tau_{s p}(y, t)$ corresponding to the second motion which becomes steady in time are presented in different forms whose equivalence is graphically proved in Figures 2 and 3. This proof, as well as the fact that known solutions from the existing literature for the incompressible Newtonian fluids are recovered as limiting cases of the present results, could be an evidence of the correctness of present results. The presentation of the starting solutions of a given motion as a sum of its steady-state and transient components is important for the experimentalists who want to eliminate the transients from their experiments.
To get some physical insight of present results, the influence of the Weissenberg number $\mathrm{We}$ and the porosity parameter $K$ on the fluid motion has been brought to light in Figures 4 and 5 . The required time to reach the steady-state for the motion which becomes steady in time is graphically determined in Figures 6 and 7 for two distinct values of We and $K$, respectively. This is the time after which the diagrams of the starting solution $u_{s}(y, t)$ superpose over those of its steady-state component $u_{s p}(y, t)$. In practice, as we previously mentioned, this time is important for those who want to eliminate the transients from their experiments. The time variations of the midplane steady-state velocity and shear stress fields are presented in Figures 8 and 9 for $K=0.5$ and 1 and different values of We.

In Figure 4, for comparison, the diagrams of the velocity fields $u(y, t)$ and $u_{N}(y, t)$ given by equalities (25) and (30), respectively, are together presented at two distinct values of the time $t$ for decreasing values of We. The profiles of the starting solution $u(y, t)$ are also presented in Figure 5 for three decreasing values of $K$. In all cases, the fluid velocity in absolute value smoothly decreases from maximum values on the lower plate to the zero value on the stationary plate. As expected, in absolute value, it is an increasing function with respect to the time $t$ and diminishes for increasing values of $K$. Consequently, as expected, in the presence of a porous medium, the fluid motion becomes more difficult. Moreover, as it results from Figure 4 , the fluid velocity $u(y, t)$ in absolute value is a decreasing function with regard to the Weissenberg number $\mathrm{We}$ and converges to the Newtonian solution $u_{N}(y, t)$ if $\mathrm{We} \longrightarrow 0$. This behavior of the motion of the Maxwell fluid can be explained by the fact that, as it 


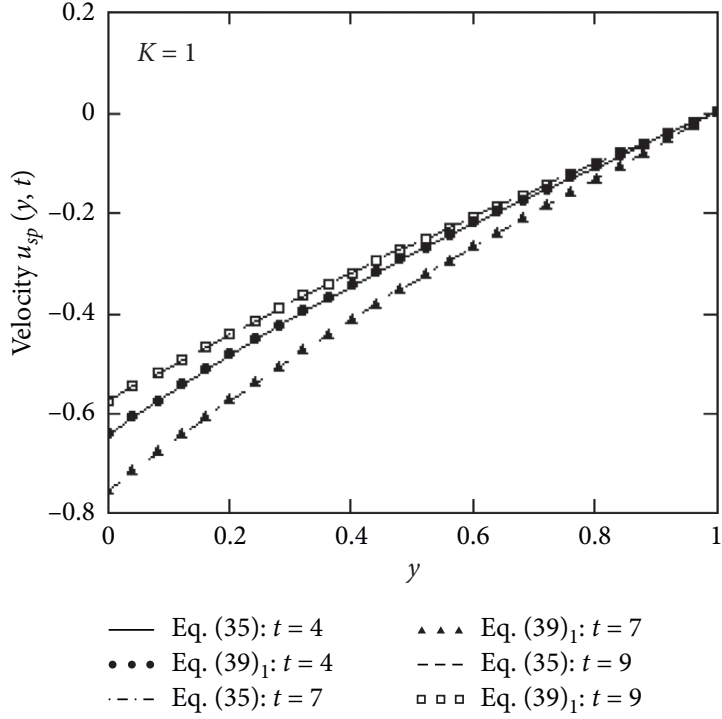

(a)

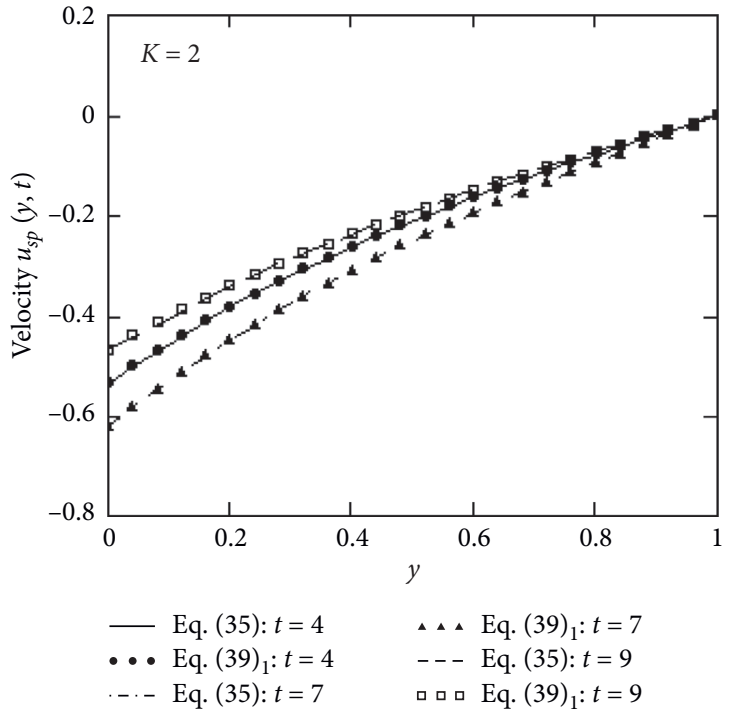

(b)

Figure 2: Profiles of the steady-state component $u_{s p}(y, t)$ given by equalities (35) and (39) for $\omega=\pi / 12$, We $=0.7, K=1$ and 2 , and three values of the time $t$.

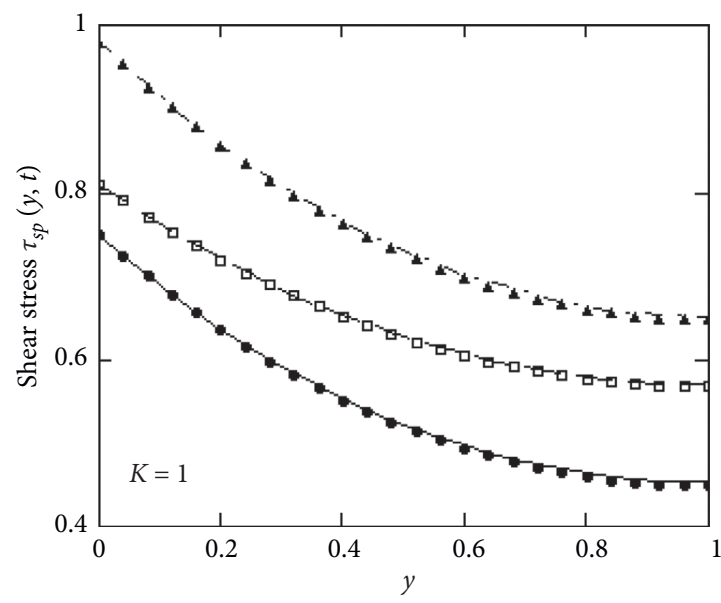

Eq. (36): $t=4$
E. Eq. $(39)_{2}: t=4$

-.. Eq. (36): $t=7$

$\triangle$ Eq. $(39)_{2}: t=7$

- - Eq. (36): $t=9$

ㅁㅁ Eq. $(39)_{2}: t=9$

(a)

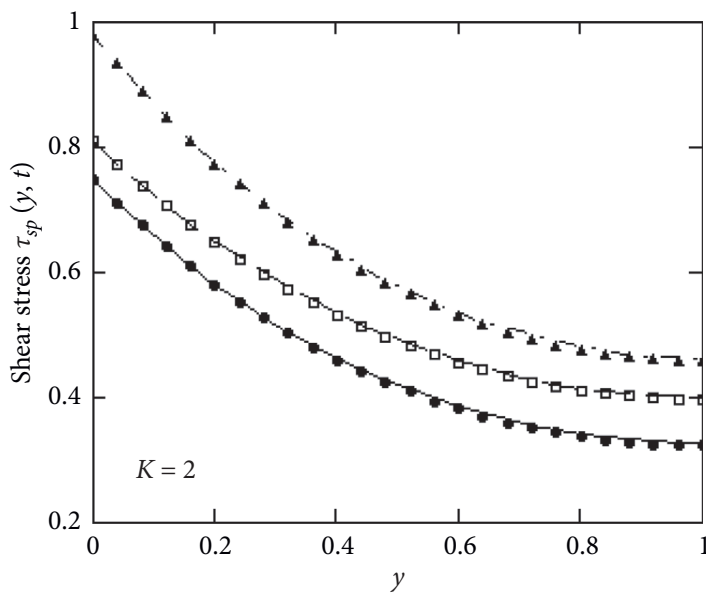

$$
\begin{aligned}
& \text { Eq. (36): } t=4 \\
& \text { - Eq. }(39)_{2}: t=4 \\
& \ldots-\text { Eq. (36): } t=7
\end{aligned}
$$

(b)

Figure 3: Profiles of the steady-state component $\tau_{s p}(y, t)$ given by equalities (34) and (39) for $\omega=\pi / 12$, We $=0.7, K=1$ and 2 , and three values of the time $t$.

was proved by Poole [26], the Weissenberg number represents the ratio of elastic forces to viscous forces. Consequently, at the same elastic properties of the fluid, an increase in We means a diminution of viscous forces and therefore an increase in the fluid velocity.

Time convergence of the starting solution $u_{s}(y, t)$ to its steady-state component $u_{s p}(y, t)$ is shown in Figures 6 and 7 for distinct values of We and $K$. From these figures, it also results that the required time to reach the steady-state for this motion that becomes permanent in time is an increasing function with regard to the Weissenberg number We and diminishes for increasing values of the porosity parameter $K$. This means that the steady-state is rather obtained for the oscillatory motion of the incompressible Newtonian fluids induced by sine oscillations of the shear stress on the boundary as compared with incompressible UCM fluids 


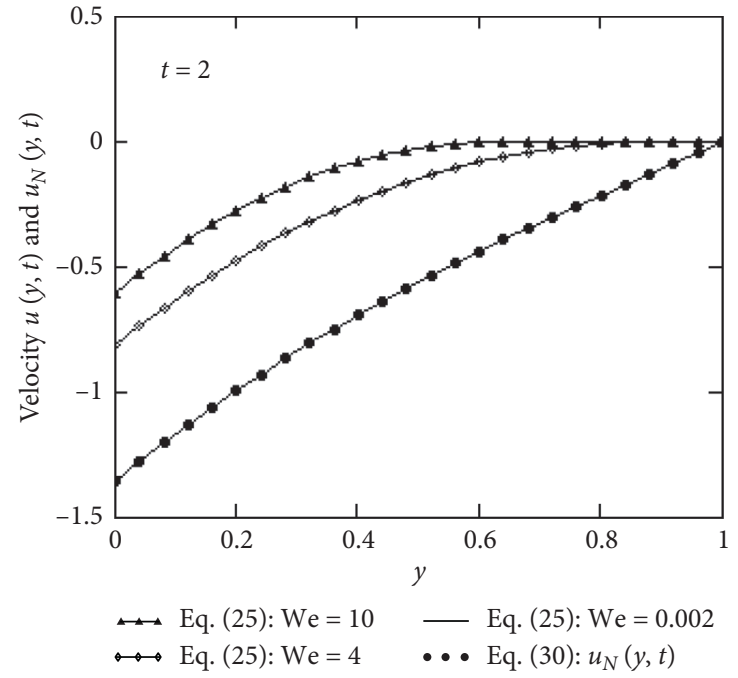

(a)

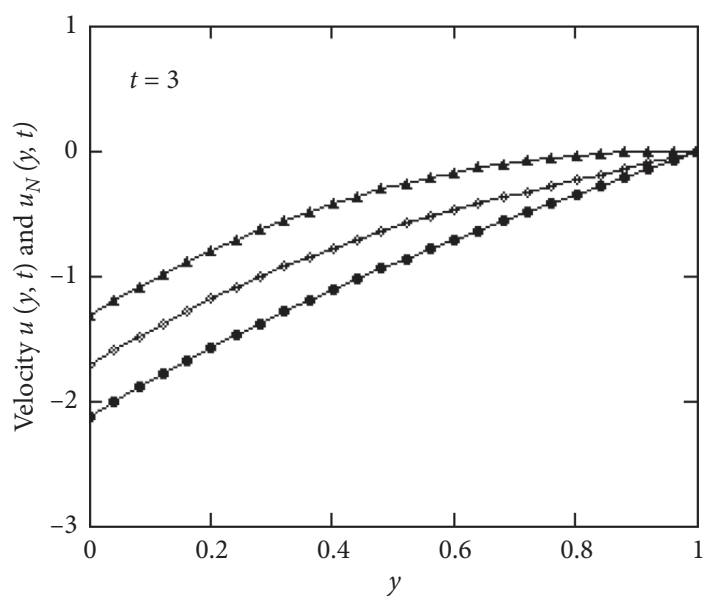

$\leftrightarrow$ Eq. (25): $\mathrm{We}=10 \longrightarrow$ Eq. (25): $\mathrm{We}=0.002$

$\leftrightarrow$ Eq. (25): $\mathrm{We}=4 \quad \cdots$ Eq. $(30): u_{N}(y, t)$

(b)

Figure 4: Variations of the velocity fields $u(y, t)$ and $u_{N}(y, t)$ given by equations (25) and (30), respectively, for $K=1, t=2$ and 3 , and three values of the Weissenberg number We.

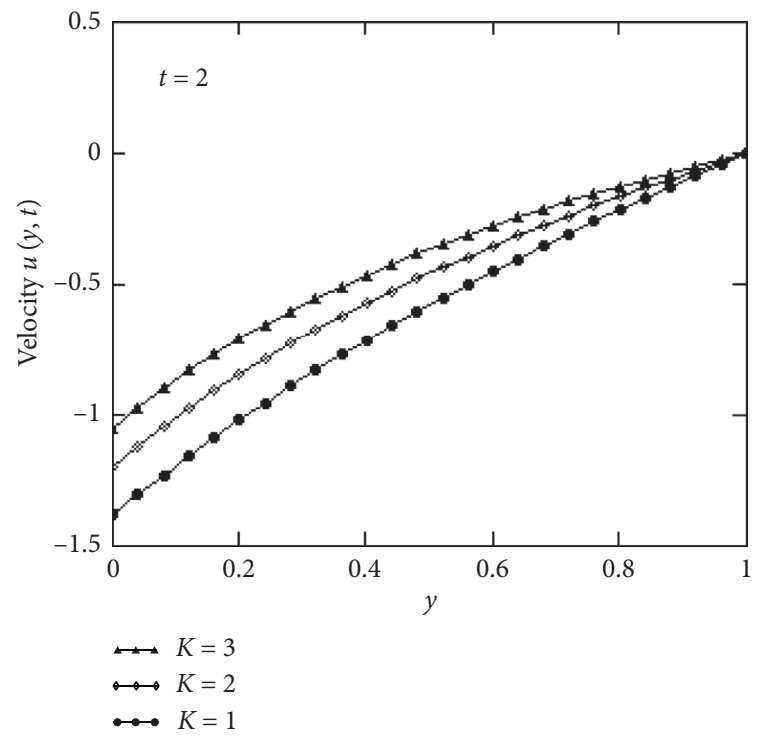

(a)

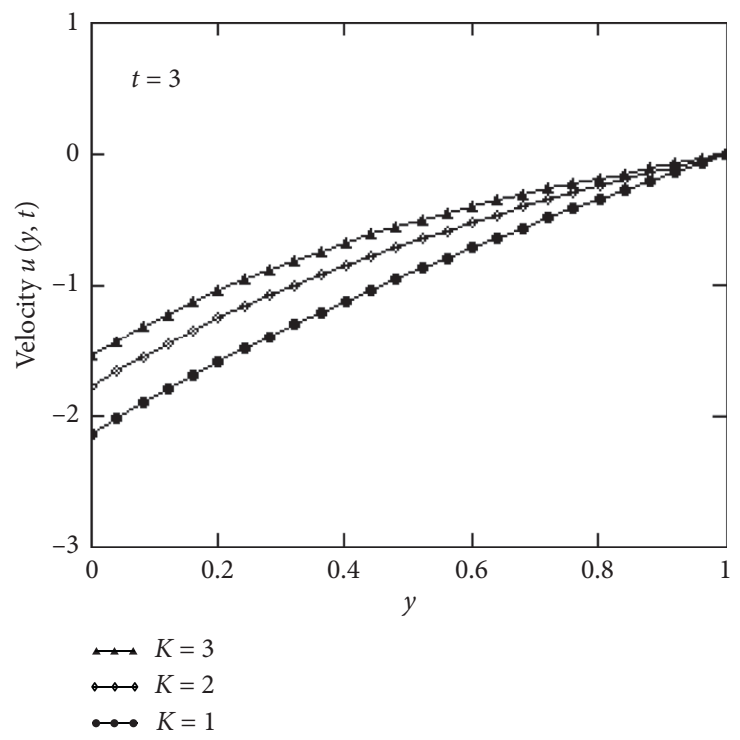

(b)

Figure 5: Variation of the velocity field $u(y, t)$ given by equality (25) for $\mathrm{We}=0.7, t=2$ and 3 , and three values of the porosity parameter $K$.

whose motion is generated by a shear stress of form (5) on the boundary. In the same time, it is clearly seen from Figure 7 that the presence of the porous medium hastens the appearance of the steady-state. The boundary condition on the upper plate is clearly satisfied.

The time variations of the midplane dimensionless steady-state velocity and shear stress fields $u_{s p}(0.5, t)$, $\tau_{s p}(0.5, t)$ and $u_{N s p}(0.5, t), \tau_{N s p}(0.5, t)$ corresponding to incompressible UCM and Newtonian fluids, respectively, are together presented in Figures 8 and 9 for $K=0.5, K=1$, and three distinct values of the Weissenberg number We. In all cases, the convergence of the non-Newtonian solutions $u_{s p}(0.5, t)$ and $\tau_{s p}(0.5, t)$ to the corresponding Newtonian solutions if $\mathrm{We} \longrightarrow 0$, as well as the oscillating specific features of the fluid motion, is clearly visualized. The amplitude of the oscillations, as expected, diminishes for increasing values of $K$ both for velocity and shear stress. In addition, the values of the velocity $u_{s p}(0.5, t)$ and those of the corresponding shear stress $\tau_{s p}(0.5, t)$ have opposite signs at each time $t$. 


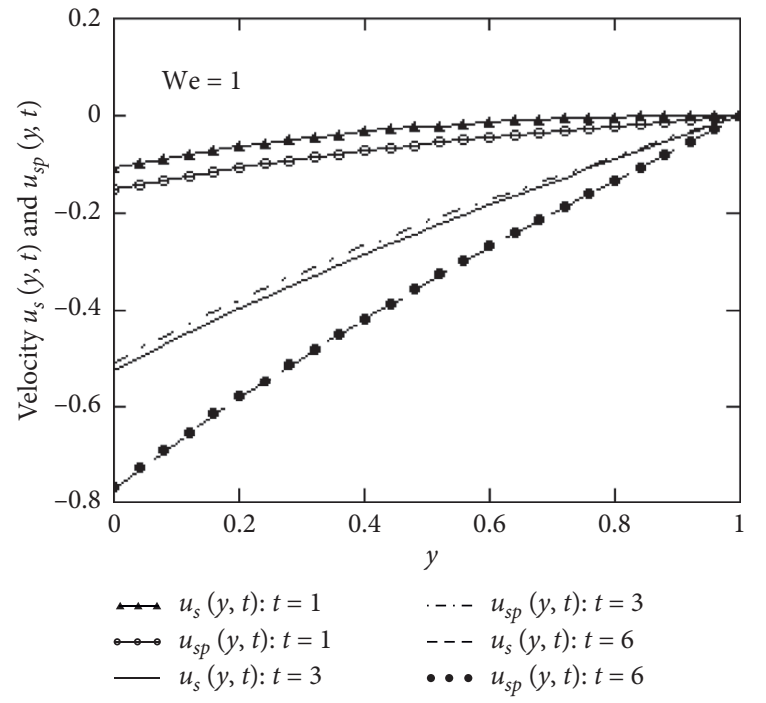

(a)

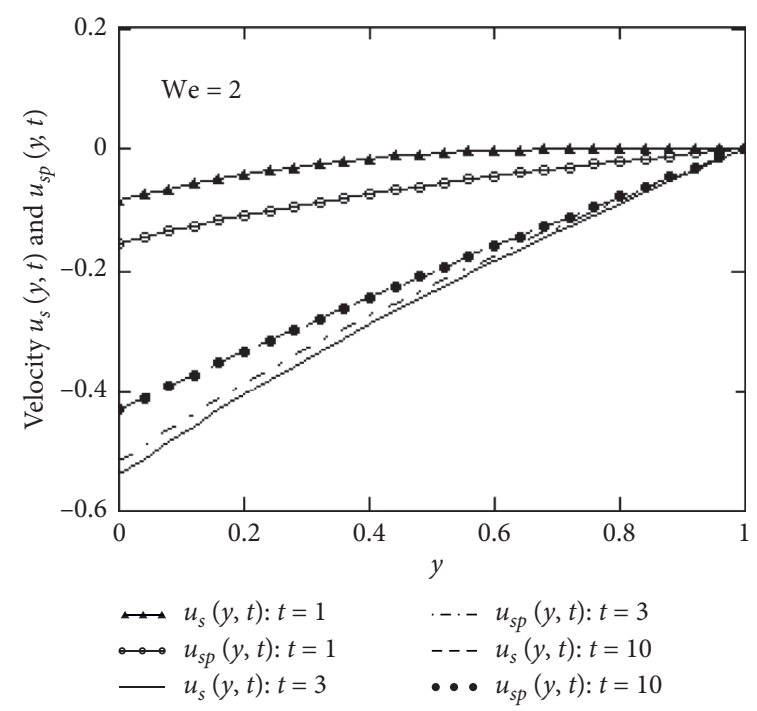

(b)

FIGURE 6: Necessary time to reach the steady-state for the motion which becomes steady in time for $\omega=\pi / 12, K=1$, and two values of the Weissenberg number We.

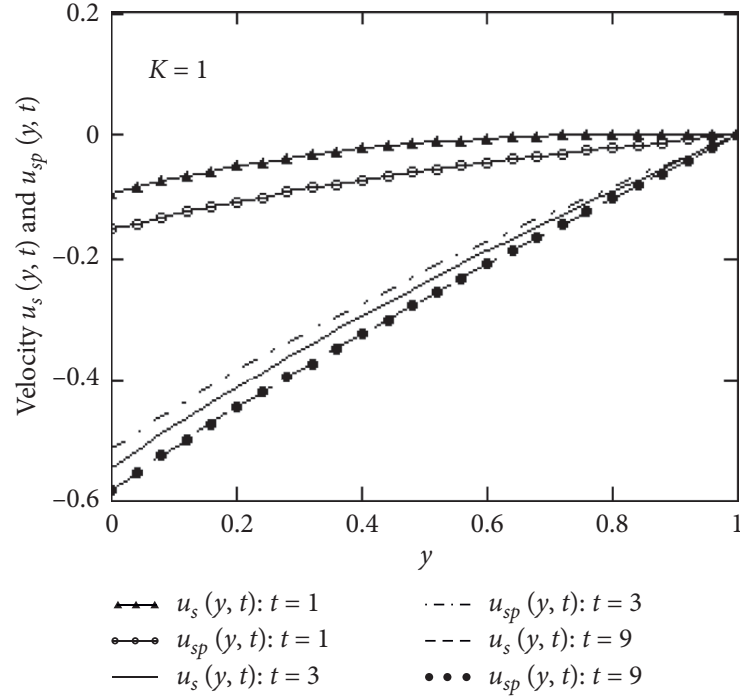

(a)

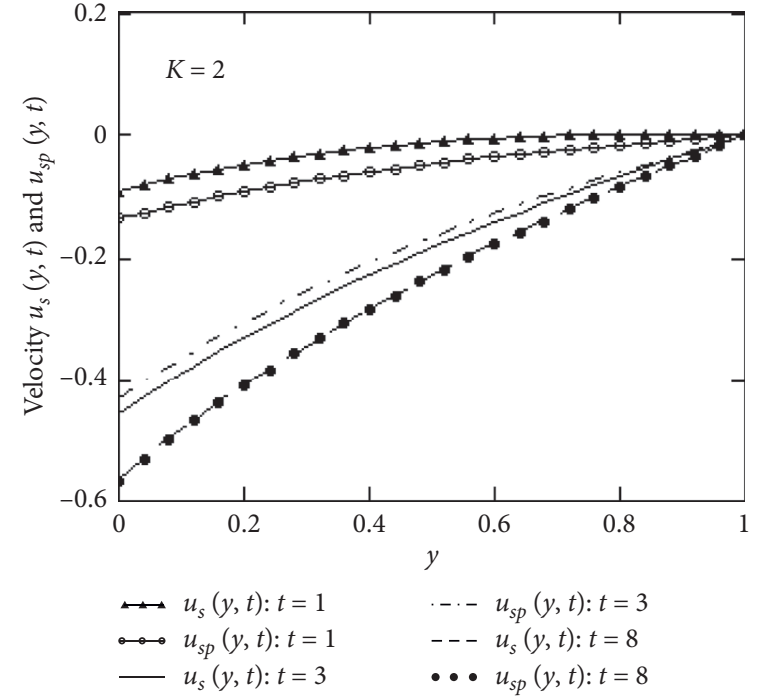

(b)

Figure 7: Necessary time to reach the steady-state for the motion which becomes steady in time for $\omega=\pi / 12$, We $=1.5$, and two values of the porosity parameter $K$. 


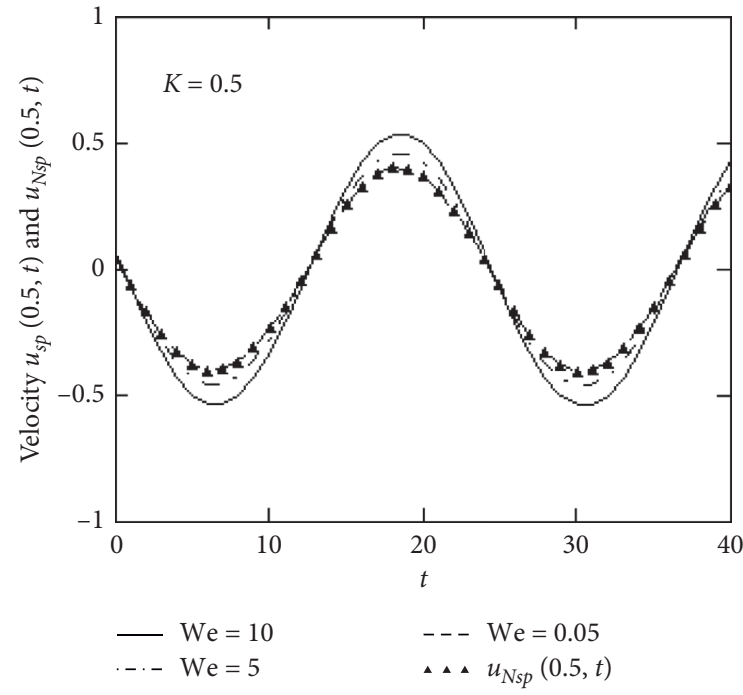

(a)

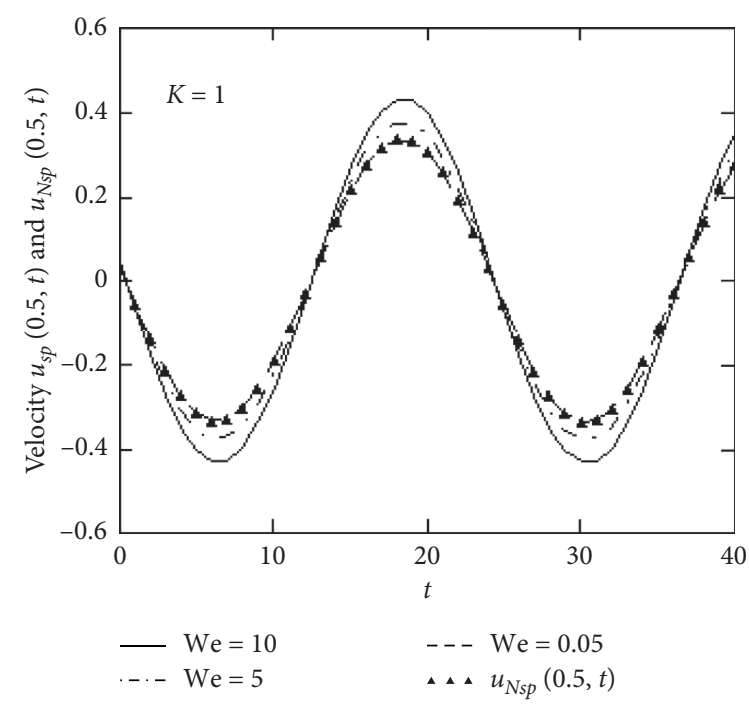

(b)

FIGURE 8: Time variations of the midplane velocities $u_{s p}(0.5, t)$ and $u_{N s p}(0.5, t)$ for $\omega=\pi / 12, K=1$ and 2 , and three values of the Weissenberg number We.

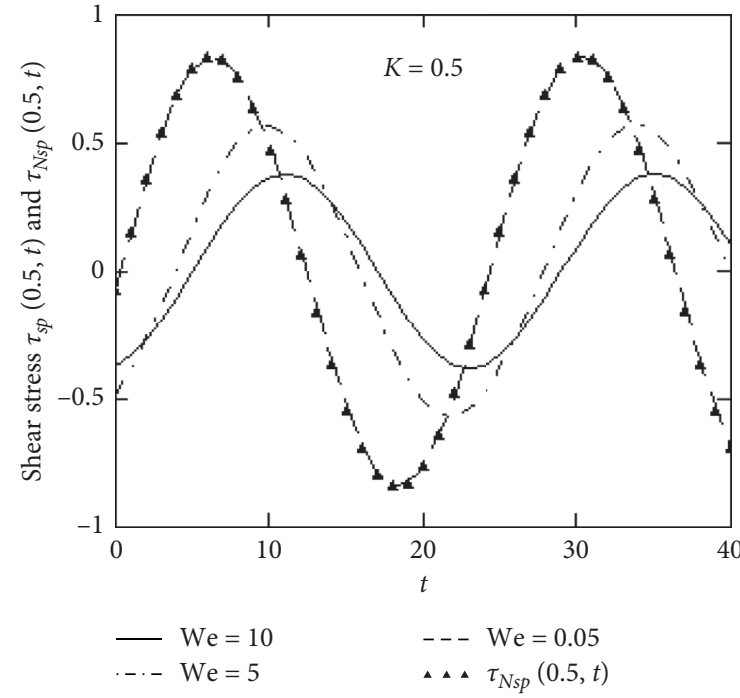

(a)

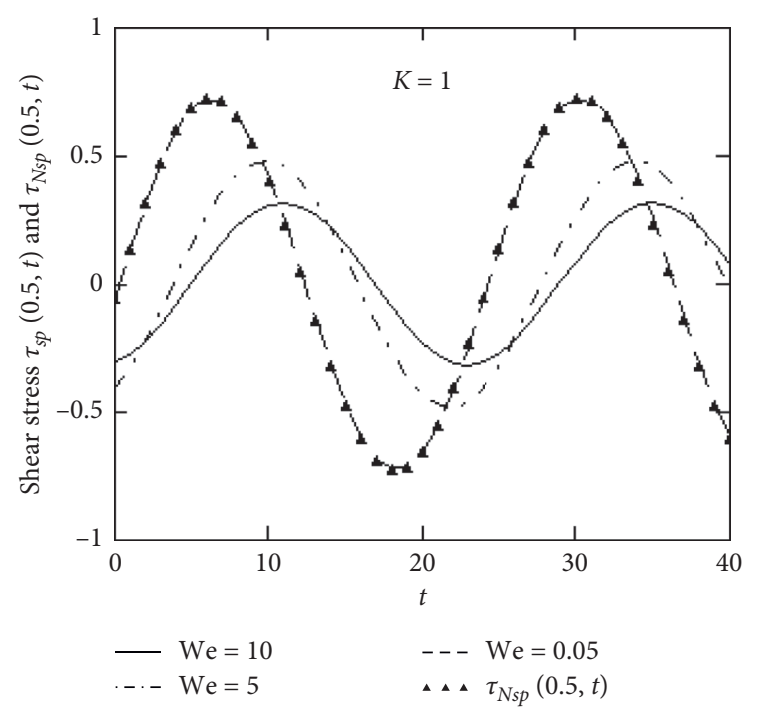

(b)

FIgURE 9: Time variations of the midplane shear stresses $\tau_{s p}(0.5, t)$ and $\tau_{N s p}(0.5, t)$ for $\omega=\pi / 12, K=0.5$ and 1 , and three values of the Weissenberg number We. 


\section{Conclusions}

Two mixed initial-boundary value problems characterizing unsteady motions of the UCM fluids between infinite parallel plates are analytically studied. Closed-form expressions are established for the dimensionless velocity and shear stress fields using the finite Fourier cosine transform. The influence of essential physical parameters on the fluid behavior was graphically investigated, and the obtained results can be such summarized:

(i) Analytical solutions for two mixed initial-boundary value problems corresponding to nonsteady motions of UCM fluids through a porous plate channel are determined.

(ii) The necessary time to touch the steady-state is graphically provided. It is an increasing function with respect to We and diminishes for increasing values of the parameter $K$.

(iii) Presence of porous medium hastens the appearance of the steady-state of the motion.

(iv) The amplitude of the oscillations corresponding to the steady-state solutions wanes in the presence of a porous medium both for the fluid velocity and the adequate shear stress.

(v) Solutions corresponding to movements of Newtonian fluids induced by ramp type or oscillatory shear stresses on the boundary are acquired as limiting cases of the present results.

\section{Nomenclature}

$\begin{array}{ll}v: & \text { Velocity field } \\ \lambda: & \text { Relaxation time } \\ u: & \text { Fluid velocity in } x \text {-direction } \\ \rho: & \text { Fluid density } \\ L: & \text { Velocity gradient } \\ \mu: & \text { Fluid viscosity } \\ T: & \text { Cauchy stress tensor } \\ \nu=\mu / \rho: & \text { Kinematic viscosity } \\ S: & \text { Extra-stress tensor } \\ \tau=S_{x y}: & \text { Shear stress } \\ k: & \text { Permeability of porous medium } \\ \sigma=S_{x x}: & \text { Normal stress } \\ p: & \text { Pressure } \\ \phi: & \text { Porosity of porous medium } \\ R: & \text { Darcy's resistance } \\ \mathrm{We}: & \text { Weissenberg number } \\ \mathbf{e}_{x}: & \text { Unit vector along the } x \text {-axis } \\ u_{F n}(t): & \text { Finite Fourier cosine transform of } u(y, t) ;\end{array}$

\section{Appendix}

$$
\begin{aligned}
v_{F n} & =\int_{0}^{1} v(y) \cos \left(\mu_{n} y\right) d y, \quad v(y)=2 \sum_{n=0}^{\infty} v_{F n} \cos \left(\mu_{n} y\right) ; \mu_{n}=\frac{(2 n+1) \pi}{2}, \\
\int_{0}^{1} \frac{d^{2} v(y)}{d y^{2}} \cos \left(\mu_{n} y\right) d y & =-\mu_{n}^{2} v_{F n}-\left.\frac{d v(y)}{d y}\right|_{y=0}+(-1)^{n} \mu_{n} v(1), \\
y & =1-2 \sum_{n=0}^{\infty} \frac{1}{\mu_{n}^{2}} \cos \left(\mu_{n} y\right) .
\end{aligned}
$$

\section{Data Availability}

No data were used to support this study.

\section{Conflicts of Interest}

The authors declare that they have no conflicts of interest regarding the publication of this paper.

\section{References}

[1] T. Hayat, R. Sajjad, Z. Abbas, M. Sajid, and A. A. Hendi, "Radiation effects on MHD flow of Maxwell fluid in a channel with porous medium," International Journal of Heat and Mass Transfer, vol. 54, no. 4, pp. 854-862, 2011.

[2] T. Hayat, R. Sajjad, A. Alsaedi, T. Muhammad, and R. Ellahi, "On squeezed flow of couple stress nanofluid between two parallel plates," Results in Physics, vol. 7, pp. 553-561, 2017.
[3] M. Renardy, "Inflow boundary conditions for steady flow of viscoelastic fluids with differential constitutive laws," Rocky Mountain Journal of Mathematics, vol. 18, no. 2, pp. 445-453, 1988.

[4] M. Renardy, "Recent advances in the mathematical theory of steady flow of viscoelastic fluids," Journal of Non-newtonian Fluid Mechanics, vol. 29, no. 1, pp. 11-24, 1988.

[5] K. R. Rajagopal, "A new development and interpretation of the Navier-Stokes fluid which reveals why the "Stokes assumption" is inapt," International Journal of Non-linear Mechanics, vol. 50, pp. 141-151, 2013.

[6] T. W. Ting, "Certain non-steady flows of second-order fluids," Archive for Rational Mechanics and Analysis, vol. 14, no. 1, pp. 1-26, 1963.

[7] N. D. Waters and M. J. King, "Unsteady flow of an elasticoviscous liquid," Rheologica Acta, vol. 9, no. 3, pp. 345-355, 1970. 
[8] R. Bandelli and K. R. Rajagopal, "Start-up flows of second grade fluids in domains with one finite dimension," International Journal of Non-linear Mechanics, vol. 30, no. 6, pp. 817-839, 1995.

[9] M. E. Erdogan, "On unsteady motions of a second-order fluid over a plane wall," International Journal of Non-linear Mechanics, vol. 38, no. 7, pp. 1045-1051, 2003.

[10] C. Fetecau, C. Fetecau, and M. Imran, "Axial Couette flow of an Oldroyd-B fluid due to a time-dependent shear stress," Mathematical Reports, vol. 11, no. 61, pp. 145-154, 2009.

[11] C. Fetecau, M. Imran, C. Fetecau, and I. Burdujan, "Helical flow of an Oldroyd-B fluid due to a circular cylinder subject to time-dependent shear stresses," Zeitschrift für angewandte Mathematik und Physik, vol. 61, no. 5, pp. 959-969, 2010.

[12] D. Vieru, C. Fetecau, and C. Fetecau, "Unsteady flow of a generalized Oldroyd-B fluid due to an infinite plate subject to a time-dependent shear stress," Canadian Journal of Physics, vol. 88, no. 9, pp. 675-687, 2010.

[13] A. U. Awan, C. Fetecau, and Q. Rubbab, "Axial Couette flow of a generalized Oldroyd-B fluid due to a longitudinal timedependent shear stress," Quaestiones Mathematicae, vol. 33, no. 4, pp. 429-441, 2010.

[14] M. Jamil, C. Fetecau, and M. Imran, "Unsteady helical flows of Oldroyd-B fluids," Communications in Nonlinear Science and Numerical Simulation, vol. 16, no. 3, pp. 1378-1386, 2011.

[15] C. Fetecau, C. Fetecau, and M. Rana, "General solutions for the unsteady flow of second-grade fluids over an infinite plate that applies arbitrary shear to the fluid," Zeitschrift für Naturforschung A, vol. 66, no. 12, pp. 753-759, 2011.

[16] M. Javaid, M. Imran, C. Fetecau, and D. Vieru, "General solutions for the mixed boundary value problem associated to hydromagnetic flows of a viscous fluid between symmetrically heated parallel plates," Thermal Science, vol. 24, no. 2 Part B, pp. 1389-1405, 2020.

[17] G. A. Danish, M. Imran, C. Fetecau, and D. Vieru, "First exact solutions for mixed boundary value problems concerning the motions of fluids with exponential dependence of viscosity on pressure," AIP Advances, vol. 10, no. 6, Article ID 065206, 2020.

[18] S. Wang, P. Li, and M. Zhao, "Analytical study of oscillatory flow of Maxwell fluid through a rectangular tube," Physics of Fluids, vol. 31, no. 6, Article ID 063102, 2019.

[19] X. Sun, S. Wang, and M. Zhao, "Oscillatory flow of Maxwell fluid in a tube of isosceles right triangular cross section," Physics of Fluids, vol. 31, no. 12, Article ID 123101, 2019.

[20] C. Fetecau, R. Ellahi, and S. M. Sait, "Mathematical analysis of Maxwell fluid flow through a porous plate channel induced by a constantly accelerating or oscillating wall," Mathematics, vol. 9, no. 1, 2021.

[21] A. Shahid, "The effectiveness of mass transfer in the MHD upper-convected Maxwell fluid flow on a stretched porous sheet near stagnation point: a numerical investigation," Inventions, vol. 5 , no. 4 , p. $64,2020$.

[22] J. G. Oldroyd, "On the formulation of rheological equations of state," Proceedings of the Royal Society of London. Series A Mathematical, Physical and Engineering Sciences, vol. 200, no. 1063 , pp. 523-541, 1950.

[23] S. Karra, V. Průša, and K. R. Rajagopal, "On Maxwell fluids with relaxation time and viscosity depending on the pressure," International Journal of Non-linear Mechanics, vol. 46, no. 6, pp. 819-827, 2011.

[24] F. Wang and J. Liu, "The first solution for the helical flow of a generalized Maxwell fluid within annulus of cylinders by new definition of transcendental function $B_{N}\left(r r_{n}\right)$," Mathematical
Problems in Engineering, vol. 2020, Article ID 8919817, 15 pages, 2020.

[25] M. Khan, R. Malik, and A. Anjum, "Exact solutions of MHD second Stokes flow of generalized Burgers fluid," Applied Mathematics and Mechanics, vol. 36, no. 2, pp. 211-224, 2015.

[26] R. J. Poole, “The deborah and Weissenberg numbers," Rheology Bulletin, vol. 53, no. 2, pp. 32-39, 2012. 